\title{
Software quality: predicting reliability of a software using decision tree
}

\begin{abstract}
System availability can be expressed as an attribute of reliability that determines the total time a system or component is functioning. Most available models try to predict availability of a software during its life cycle but there are very few or no models that predict a software going days without a failure. Over the years, decision tree model have been used as a reliable technique for prediction. In this study, based on the sample data collected by John Musa of Bell Telephone Laboratories, a decision tree model has been used to predict the availability of a system going days without a failure. This study concluded that a decision tree model is able to decide availability of a software in terms of going days without a failure.
\end{abstract}

Keyword: Software quality; Software measurement; Software realibility; Software availability; Decision tree model 